\title{
NUEVOS INDICADORES SOCIOAMBIENTALES PARA LOS REPORTES DE SOSTENIBILIDAD. CASO: MINERAS PERUANAS EN LA BOLSA DE VALORES DE LIMA
}

\author{
NEW SOCIO-ENVIRONMENTAL INDICATORS FOR SUSTAINABILITY REPORTS. \\ CASE: PERUVIAN MINING COMPANIES IN LIMA STOCK EXCHANGE
}

Brandelt Jesus Astorga de la Cruz Universidad del Pacífico Lima, Perú

ORCID: https://orcid.org/0000-0002-7674-4140

Correo electrónico: jesus.astorgad@gmail.com

\section{RESUMEN}

Objetivo: Diseñar indicadores complementarios socioambientales de generación de valor como parte de los Reportes de Sostenibilidad para las empresas mineras peruanas que cotizaron en la Bolsa de Valores de Lima en el año 2017. Método: El estudio tuvo un alcance exploratorio y descriptivo, y un enfoque mixto: cualitativo y cuantitativo. El diseño de la investigación fue de tipo transversal y utilizó un muestreo no probabilístico, por conveniencia del investigador. Resultados: Los resultados del trabajo de investigación aportan nuevos indicadores para las empresas mineras del Perú. Así, se proponen los siguientes indicadores sociales como: la relación entre el índice de frecuencia de accidentes y los ingresos; la relación de participación ciudadana y número de quejas registradas; el porcentaje de horas de formación vinculadas con la prevención de accidentes; y la relación entre el canon minero y los ingresos. Relativo a los indicadores ambientales, se proponen los siguientes: porcentaje del agua reciclada frente al total de agua utilizada, y porcentaje de residuos peligrosos frente al total de residuos. Conclusiones: Se concluyó que los indicadores complementarios sugeridos podrían contribuir a la generación de valor de las empresas mineras del Perú.

Palabras clave: Socioambiental; indicadores; minería; reportes; sostenibilidad.

\begin{abstract}
Objective: Design complementary socio-environmental indicators of generation value as part of the Sustainability Reports for Peruvian mining companies listed on the Lima Stock Exchange in 2017. Method: The study had an exploratory and descriptive scope, and a mixed approach: qualitative and quantitative. The research design was cross-sectional and non-probabilistic sampling were used, for convenience of the researcher. Results: The research results bring new indicators for Peru mining companies. Thus, the following social indicators are proposed such as: the relationship between the accident frequency index and income; the relationship of citizen participation and registries number of complaints; the percentage of training hours linked to accident prevention; and the relationship between the mining tax and income. Regard to environmental indicators, the following are proposed: recycled water percentage versus the total water used, and hazardous waste percentage versus total waste. Conclusions: It concluded that the suggested complementary indicators could contribute to the generation value of Peru's mining companies.
\end{abstract}

Keywords: Socio-environmental; indicators; mining; reports; sustainability. 


\section{INTRODUCCIÓN}

Los fundamentos filosóficos o epistemológicos del presente artículo están sustentados en la doctrina del racionalismo o producción científica tecnológica sostenida por Mario Bunge (Nicomedes, 2018). De este modo, se toma como referencia el desarrollo del conocimiento desde una rama importante de la filosofía denominada Teoría del conocimiento, que puede ser abordada desde la doctrina filosófica racionalista evolucionista, cuya aplicación se da en las ciencias puras, como es el caso de las ciencias empresariales. Estas ciencias surgieron a partir de la Revolución Industrial, ante la necesidad de administrar eficiente y eficazmente diversas unidades de negocio y el uso de sus recursos como mano de obra, materias primas, entre otros (Najmaei y Sadeghinejad, 2014).

Asimismo, las ciencias empresariales han sido legitimadas en el campo de la ciencia al aportar y sugerir diversas teorías a diferentes áreas de estudio. En efecto, han ayudado a diseñar diversas teorías desde áreas como el marketing, la contabilidad, los negocios internacionales, las finanzas empresariales, entre otras (Najmaei y Sadeghinejad, 2014). Asimismo, la orientación filosófica de estas ciencias se ha caracterizado por ser pragmática, es decir, la teoría es extraída de la práctica (por ejemplo, de las empresas), y esta a su vez, se aplica de nuevo a la práctica (al mundo empresarial) (Stanford Encyclopedia of Philosophy, 2013).

El artículo contribuye a uno de los temas más relevantes de las ciencias empresariales: la responsabilidad social empresarial (RSE), a través de la propuesta de indicadores que evalúan el impacto social y ambiental por parte de las empresas mineras peruanas. Notablemente, el avance del sector minero presenta ventajas y desventajas en el progreso de las naciones, así como también en su economía. Entre sus ventajas se encuentran la creación de empleo y el desarrollo económico, mientras que entre sus desventajas están los impactos negativos en el ámbito social y ambiental (PricewaterhouseCoopers, 2019).

Actualmente, se desarrolla la RSE como el concepto que considera la relación entre la sostenibilidad de las empresas y el lugar donde tiene sus operaciones. Entre los instrumentos de gestión más empleados de la RSE se encuentran las Memorias de Sostenibilidad o también conocidas como los Reportes de Sostenibilidad (RS), dicha herramienta tiene como función la comunicación de los impactos de las empresas en sus operaciones (Correa et al., 2016). En ese sentido, KPMG International es una empresa perteneciente a las cuatro firmas de auditoría más grande del mundo, que clasifica estos impactos en las dimensiones: económica, social y ambiental (KPMG, 2017). Asimismo, entre las ventajas que presentan los RS se encuentran la mejora de la imagen pública, la liquidez de los títulos valores y la disminución del costo de capital (Robbins y Coulter, 2009).

Según KPMG (2017), las empresas que emiten su RS bajo los lineamientos del Global Reporting Initiative (GRI) se han caracterizado por constituir la Top 100 organizaciones de las principales economías del mundo. Interesantemente, las empresas peruanas, sobre todo aquellas del sector minero, que publican sus RS con la metodología GRI han experimentado un menor desempeño económico en los últimos años lo que ha limitado el desarrollo de sus operaciones (PricewaterhouseCoopers, 2019). Esto puede ser la causa de que los RS de estas empresas mineras no estén brindando información óptima del sector a sus stakeholders. Se define stakeholders quienes son las partes interesadas a los diferentes grupos de personas que influyen en una empresa (Correa et al., 2016).

En efecto, los continuos conflictos de tipo socioambiental que se desarrollan vinculados al sector minero se consideran como otro mecanismo que influye en el impedimento del progreso del sector. Instituciones como el Ministerio de Energía y Minas (2017) y la Defensoría del Pueblo (2017) asignan en sus memorias anuales, a partir del 2010 a la actualidad, un mayor contenido relacionado a los conflictos socioambientales en el sector minero, dado su papel relevante en dicho sector. Autores como Briano (2014) y Hernández (2016) indican que los instrumentos de gestión como los RS podrían impactar positivamente en la mitigación de los conflictos. En esa línea, autores como Correa et al. (2016) concuerdan en que diseñar indicadores con distintos criterios referentes a las dimensiones socioambientales contribuirán a resolver problemas de tal índole. Adicionalmente, la generación de estos indicadores complementarios debe estar basada en una metodología que permita su comparabilidad, como es el caso de los lineamientos propuestos por el GRI (Correa et al., 2016).

En efecto, se encontró estudios que formulan indicadores complementarios tomando como marco de referencia al GRI, pero ninguno se centró en el sector minero, ni analizó los indicadores desde la perspectiva de un país en vías de desarrollo, como es el caso del Perú. De esta manera, se demuestra la existencia de la falta de literatura en referencia al planteamiento de indicadores complementarios bajo el marco GRI para el sector minero en un país emergente. En ese marco, el objetivo del estudio fue plantear indicadores complementarios que contribuyan a la generación de valor para los grupos de interés en el sector minero.

Como resultado, el estudio propone seis nuevos indicadores complementarios que las empresas mineras peruanas deberían considerar en sus RS están clasificados en las 
dimensiones social y ambiental, con cuatro y dos indicadores, respectivamente. Finalmente, una vez abordado y contextualizado la relevancia que representa el tema del estudio se continuará con el siguiente acápite.

\section{MATERIALES Y MÉTODOS}

El estudio fue de tipo cuantitativo no experimental y de corte transversal; realizado durante el periodo 2017. Asimismo, la investigación tuvo un alcance exploratorio y descriptivo. Exploratorio, ya que examina un tema poco estudiado. En ese sentido, es importante recordar lo explicado en la introducción, referente a la inexistencia de literatura científica que proponga indicadores complementarios bajo el marco GRI que, al mismo tiempo, permitan una mayor fundamentación y presentación óptima con el objetivo de atender las necesidades del sector minero.

Para efectos del estudio, el sistema estadístico abordado fue la estadística descriptiva. En tal sentido, se midieron las variables que conformaron cada uno de los indicadores; por ejemplo, se propuso el indicador relación entre el índice de frecuencia de accidentes y los ingresos. Luego, tales variables fueron medidas para la muestra de empresas mineras examinadas.

Respecto a la selección de la muestra del estudio, se consideró un muestreo no probabilístico por conveniencia del investigador. Ello debido a que el estudio busca cumplir con las siguientes características: que las empresas mineras peruanas que coticen en la Bolsa de Valores de Lima (BVL); que comuniquen sus reportes de sostenibilidad comparables; y que representen más del $50 \%$ de las ventas del sector minero en los últimos cinco años (2013-2017). Adicionalmente, el procesamiento de datos y elaboración de instrumentos descriptivos, como los gráficos de barras que resumieron la información presentada, se realizó con el software SPSS Statistical Software 25.0.

Como resultado, son cuatro las empresas mineras peruanas más importantes; entre ellas están: Cerro Verde, Buenaventura, Minsur y Volcan. Dichas empresas representan el 50,9\% del total de ventas del sector minero peruano, entre el periodo 2013-2017, que cotizaron en la BVL y que cuentan con RS comparables.

Asimismo, atendiendo a las características del estudio, se procedió considerando la siguiente estructura: En primer lugar, se determinaron los aspectos por tratar en las dimensiones sociales y ambientales de las empresas mineras del Perú. Con este fin, se establecen dos criterios: los temas materiales comunicados en los Reportes de Sostenibilidad (RS) de Cerro Verde, Minsur y Volcan; y los problemas socioambientales más importantes del sector minero peruano en el 2017, según los estudios de Brereton et al. (2018) y la Defensoría del Pueblo (2017).

En segundo lugar, se continuó con la identificación de indicadores en los aspectos sociales y ambientales de las cuatro empresas mineras peruanas sobresalientes. Para ello, se preparó un cuadro de revisión para determinar plenamente los indicadores registrados bajo el sistema GRI en los aspectos sociales y ambientales de Buenaventura, Minsur, Cerro Verde y Volcan.

En tercer lugar, se prosiguió con la formulación de los indicadores complementarios para las dimensiones sociales y ambientales de las empresas mineras peruanas. Es importante señalar que la obtención de los indicadores implica el seguimiento de las fases anteriores. Por último, se realizó un análisis enfocado en la generación de valor e impacto en la sostenibilidad de la relación entre las variables que componen los indicadores complementarios propuestos. A continuación, se presentan los resultados obtenidos.

\section{RESULTADOS}

La tabla 1 muestra los seis indicadores complementarios socioambientales propuestos por el estudio, algunos de los cuales pueden ser calculados con la información que se dispone de las empresas pertenecientes a la muestra, a modo de proporcionar indicadores complementarios que puedan ser considerados en sector minero.

A continuación, se procede con el análisis de indicadores en sus respectivas dimensiones: social y ambiental.

La dimensión social está vinculada al conjunto de reglas de convivencia que se establecen para la regulación de las conductas de las personas que interactúan dentro de un sistema general (Correa et al., 2016). Asimismo, el Ministerio de Energía y Minas (2017) exige a las empresas mineras velar por los impactos que puedan ocasionar sus operaciones, de manera que permitan la administración y prevención de conflictos socioambientales. A continuación, se describirán los resultados encontrados de los indicadores de la dimensión social.

Indicador 1: Relación entre el índice de frecuencia de accidentes y los ingresos

Según Salim (2014), el trabajo en el sector minero se considera de alto riesgo debido al nivel de mortalidad en este sector. Ello se evidencia en el Anuario Minero del Ministerio de Energía y Minas (2017), en el cual se menciona que el índice de frecuencia de accidentes es relevante para las empresas mineras peruanas. En esa línea, Salim, en su estudio, encontró que las empresas que generan valor para sus stakeholders son aquellas que tienen mayores ingresos 
Tabla 1

Indicadores complementarios que muestran el grado de relación entre la información mostrada en los RS y la generación de valor de las empresas mineras

\begin{tabular}{|c|c|c|c|}
\hline Dimensión & Temas relevantes & Indicador propuesto & Forma de cálculo \\
\hline \multirow[t]{2}{*}{ Social } & \multirow[t]{2}{*}{$\begin{array}{l}\text { Salud y seguridad en el } \\
\text { trabajo }\end{array}$} & \multirow[t]{2}{*}{$\begin{array}{l}\text { Relación entre el índice de frecuencia de } \\
\text { accidentes y los ingresos }\end{array}$} & $\begin{array}{l}\text { Índice de frencuencia } \\
\text { de accidentes }\end{array}$ \\
\hline & & & Ingresos \\
\hline \multirow[t]{2}{*}{ Social } & \multirow[t]{2}{*}{ Comunidades locales } & \multirow{2}{*}{$\begin{array}{l}\text { Relación entre la participación ciudadana y el } \\
\text { número de quejas registradas }\end{array}$} & $\begin{array}{c}\text { Mecanismos de particpación } \\
\text { ciudadana }\end{array}$ \\
\hline & & & $\overline{\text { Número de quejas registradas }}$ \\
\hline \multirow[t]{2}{*}{ Social } & \multirow[t]{2}{*}{ Formación y enseñanza } & \multirow{2}{*}{$\begin{array}{l}\text { Porcentaje de horas de formación vinculadas } \\
\text { con la prevención de accidentes frente al total } \\
\text { de horas de formación }\end{array}$} & $\begin{array}{l}\text { Horas de formación vinculadas } \\
\text { a la prevención de accidentes }\end{array}$ \\
\hline & & & Total de horas de formación \\
\hline \multirow{2}{*}{ Social } & \multirow{2}{*}{$\begin{array}{l}\text { Cumplimiento } \\
\text { Socioeconómico }\end{array}$} & \multirow{2}{*}{ Relación del canon minero y los ingresos } & Canon minero \\
\hline & & & Ingresos \\
\hline \multirow{2}{*}{ Ambiental } & \multirow{2}{*}{ Agua } & \multirow{2}{*}{$\begin{array}{l}\text { Porcentaje del agua reciclada frente al total de } \\
\text { agua utilizada }\end{array}$} & Cantidad de agua reciclada \\
\hline & & & Total de agua utilizada \\
\hline Ambiental & Efluentes y residuos & $\begin{array}{l}\text { Porcentaje de residuos peligrosos frente al } \\
\text { total de residuos }\end{array}$ & $\begin{array}{c}\text { Residuos peligrosos en } \\
\text { toneladas } \\
\text { Total de residuos generados en } \\
\text { toneladas }\end{array}$ \\
\hline
\end{tabular}

Fuente: Elaboración propia

y un menor índice de frecuencia de accidentes. Como resultado se concibe el siguiente indicador:

Fórmula 1: Relación entre el índice de frecuencia de accidentes (IFA) y los ingresos.

$$
\begin{aligned}
& \begin{array}{l}
\text { Índice de frencuencia } \\
\text { de accidentes }
\end{array} \\
& \text { Ingresos }
\end{aligned}
$$

La figura 1 muestra que la empresa Cerro Verde es la minera que presenta mayor ingreso en comparación con las demás y, al mismo tiempo, presenta un bajo nivel en el índice de frecuencia de accidentes; a diferencia de Minsur, que posee un resultado menos favorable. Por lo tanto, en base a los resultados encontrados se puede considerar que Cerro Verde está generando mayor valor en la dimensión social para sus stakeholders, debido a la mejor gestión de la frecuencia de accidentes en sus operaciones.

Indicador 2: Mecanismo de participación ciudadana y número de quejas registradas

En particular, en la minería, la participación de los ciudadanos es un proceso por el cual las personas comunican sus deseos e inquietudes a las empresas. En esa línea, los mecanismos de participación ciudadana están compuestos por: un buzón de sugerencias, un monitoreo participativo del medio ambiente, agencias de información permanente, visitas a plantas de producción y una distribución de materiales informativos. La participación ciudadana también incluye información relacionada con el sector, las estrategias, los medios, entre otros aspectos, para crear un impacto en la sostenibilidad de la comunidad y de la empresa. En efecto, Alfaro y Gómez (2016), por su parte, coinciden en que mantener un seguimiento cuantitativo de temas relevantes, como el número de quejas, crea un valor social, ya que contribuye directamente a mejorar la comunicación y las relaciones entre ciudadanos y organizaciones. Teniendo en cuenta este hecho y la necesidad de comunicar los impactos de las empresas con la sostenibilidad a los stakeholders en el sector minero, se ha planteado el siguiente indicador:

La figura 2 evidencia un 5,5 en promedio de mecanismos de participación de los ciudadanos para las empresas analizadas. Por su parte, la empresa minera Volcan tiene un mayor número de quejas frente a Cerro Verde. Adicionalmente, se puede afirmar que Cerro Verde, Minsur y Volcan generan 


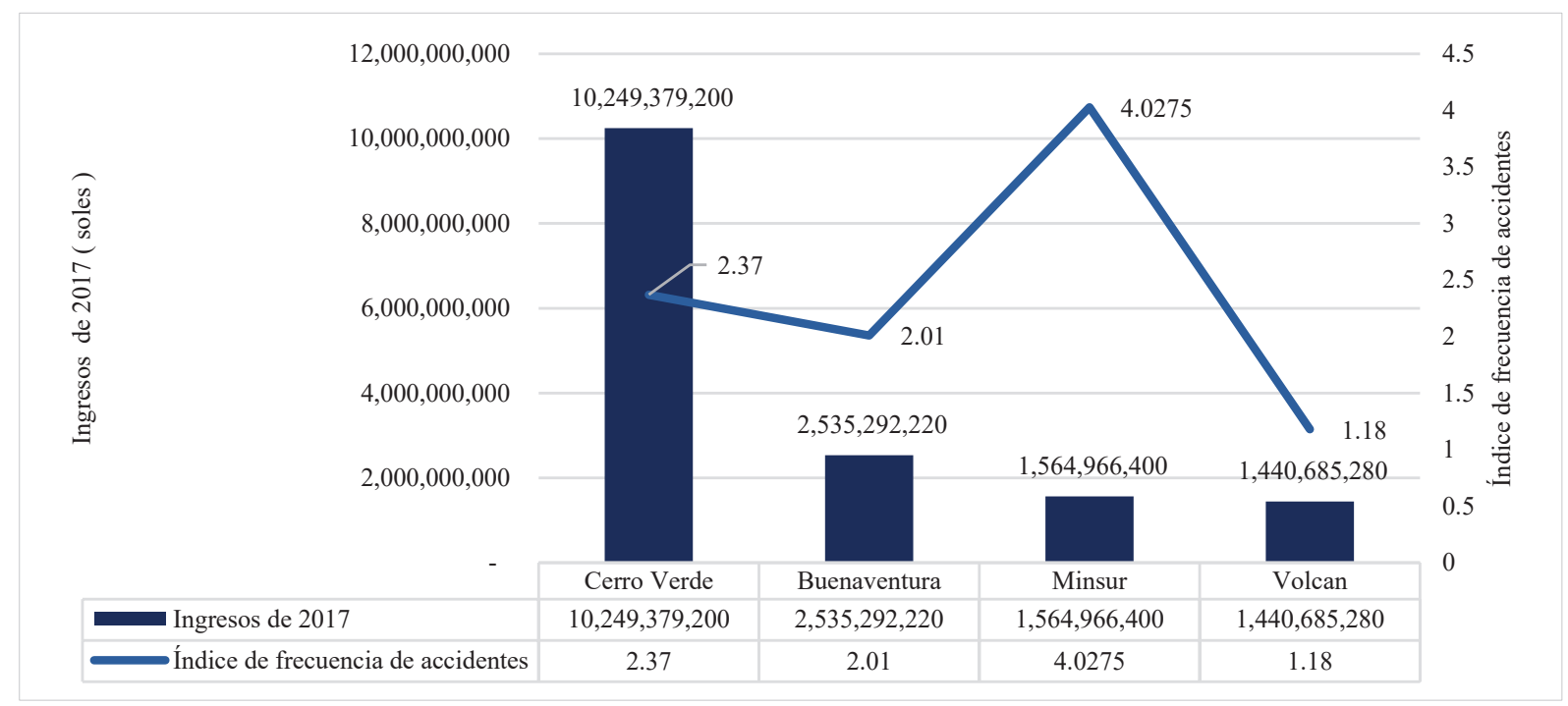

Figura 1. Relación entre el índice de frecuencia de accidentes y los ingresos de 2017 de las empresas analizadas.

Fuente: Reportes de sostenibilidad de Cerro Verde, Buenaventura, Minsur y Volcan. Database GRI, 2018 (https://database.globalreporting.org/)

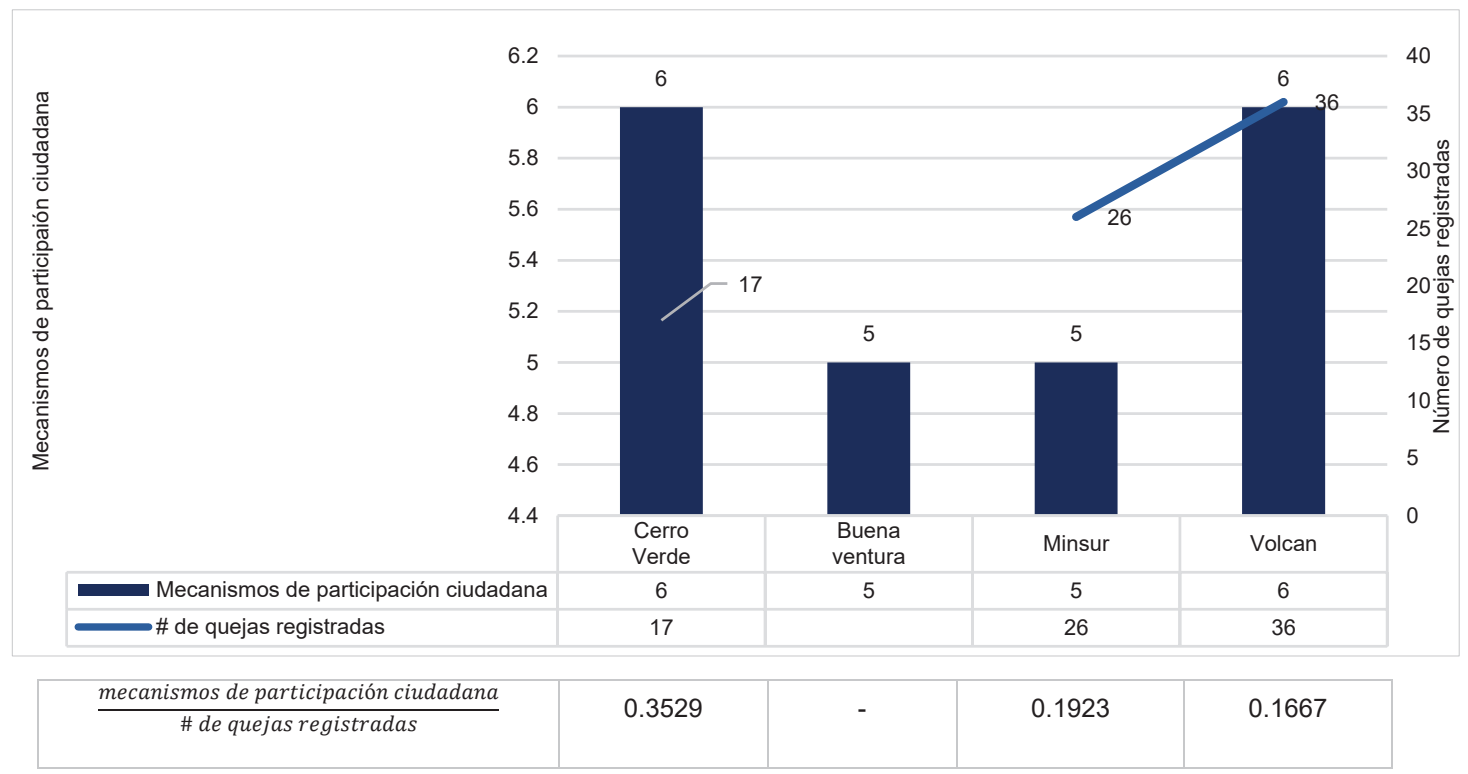

Figura 2. Distribución del mecanismo de participación ciudadana y número de quejas registradas.

Fuente: Reportes de sostenibilidad de Cerro Verde, Buenaventura, Minsur y Volcan. Database GRI, 2018 (https://database.globalreporting.org/)

un mayor valor a sus stakeholders a diferencia de Buenaventura, debido a que las empresas comunican información vinculada a temas importantes para el sector que permite la generación de valor, así como también refleja lo suficiencia para reconocer, registrar y atender las demandas que se les presentan a las compañías.
Indicador 3: Porcentaje de horas de formación vinculadas con la prevención de accidentes

El desarrollo de las ventajas competitivas en las empresas está impulsado por el capital intelectual y la formación de los trabajadores. Las horas de formación mejoran la 
productividad de los colaboradores, lo que a su vez tendría un efecto en la mejora de los resultados financieros o el aumento de los beneficios para las empresas (Organización Internacional del Trabajo, 2016).

En efecto, las horas de formación asociadas a una cultura de prevención puede ayudar a proteger la vida, el bienestar de los colaboradores y mejorar la eficiencia de la empresa para evitar problemas de cumplimiento y asegurar la adecuación del capital humano en la empresa (Guevara, 2015). De este modo, el tiempo de formación preventiva creará un mayor valor para los accionistas mediante la adquisición de conocimientos especializados para garantizar el desarrollo operacional de los colaboradores y mejorar el bienestar; razón por la cual, se plantea el siguiente indicador:

Fórmula 3: Porcentaje de horas de formación vinculado con la prevención de accidentes frente al total de horas Horas de formación vinculadas a la prevención de accidentes de formación

El presente indicador no se puede medir debido a la limitación de obtención de la información de los RS en las empresas analizadas. Ello no impide explicar los posibles resultados que se pueden obtener a partir del indicador planteado, cuyo resultado se puede encontrar entre 0 y 1 . Si el indicador es 0 , reflejaría que la empresa presenta un desinterés por los temas relacionados a la prevención de accidentes. Por otro lado, si el valor es 1, reflejaría un desinterés por otros temas diferentes a las capacitaciones.

Indicador 4: Canon minero y los ingresos

Los ingresos de una nación o de un lugar en particular ayudarán al Índice de Desarrollo Humano (IDH) en la medida en que los recursos se invierten en servicios públicos (Programa de las Naciones Unidas para el Desarrollo, 2014). En particular, el canon minero favorece al aumento de los ingresos de una zona o localidad y, por lo tanto, al desarrollo humano vital de estas personas. De este modo, una gestión adecuada del canon daría lugar a la creación de valor de las empresas y de sus actividades, a partir de lo cual se obtiene, principalmente, una licencia social que generara valor para accionistas, trabajadores y otras partes interesadas (Velarde, 2018). Al ver la necesidad de evaluar la transparencia con respecto a los ingresos, se evalúa el siguiente indicador:

Fórmula 4: Relación del canon minero y los ingresos

\section{Canon minero}

De forma similar, el resultado del indicador 4 puede presentar valores entre 0 y 1 . En el caso de resultar 0 , se reflejaría que el indicador es desfavorable para la empresa minera, pues se demostraría el desinterés de la empresa por asignar importación al canon minero. De forma similar, el valor 1 sería un indicador no favorable, ya que evidenciaría que la empresa no toma en cuenta el balance al distribuir sus beneficios. Una vez analizados los indicadores propuestos para la dimensión social, se prosigue con el análisis de los indicadores relevantes desde un plano ambiental.

\section{Dimensión ambiental}

La dimensión ambiental se relaciona con el conocimiento sobre biodiversidad. En efecto, dicha dimensión es importante para las organizaciones, ya que crean valor para sí mismas y sus stakeholders. A continuación, se define cada uno de los indicadores complementarios perteneciente a la dimensión ambiental.

Indicador 5: Porcentaje del agua reciclada frente al total de agua utilizada

Brereton et al. (2018) coinciden en que un aspecto relevante a mejorar en el sector minero peruano está relacionado con la proporción de información sobre la cantidad de agua que extraen en sus operaciones. En efecto, la producción y comunicación de políticas de agua reciclada forma parte de la eficiencia organizacional de las empresas que incorporan soluciones medioambientales en sus procesos, lo cual genera valor para los stakeholders (Striedinger, 2014). Esto complementa los resultados preliminares sobre los aspectos importantes de la minería, en los cuales se encontró que el tema relacionado al agua era crucial para las empresas mineras peruanas (Ministerio de Energía y Minas, 2017).

Considerando lo mencionado, la medición del porcentaje de uso de agua reciclada utilizada en las operaciones de las empresas mineras se determinó como la base para establecer la siguiente fórmula:

\section{Fórmula 5: Porcentaje $\begin{array}{ll}\text { del agua reciclada frente } & \text { Cantidad de agua reciclada } \\ \text { al total de agua utilizada } & \text { Total de agua utilizada }\end{array}$ al total de agua utilizada}

En cuanto a los resultados del indicador 5 mostrados en la figura 3, la empresa Buenaventura presenta un mejor resultado vinculado al uso del agua reciclada en sus actividades. Asimismo, las empresas que le siguen son Cerro Verde y Minsur. Lamentablemente, se encontró que Volcan no brinda información referente al indicador $y$, por ende, se podría deducir que no recicla el agua en sus operaciones o no tiene los recursos para medir y comunicar. 


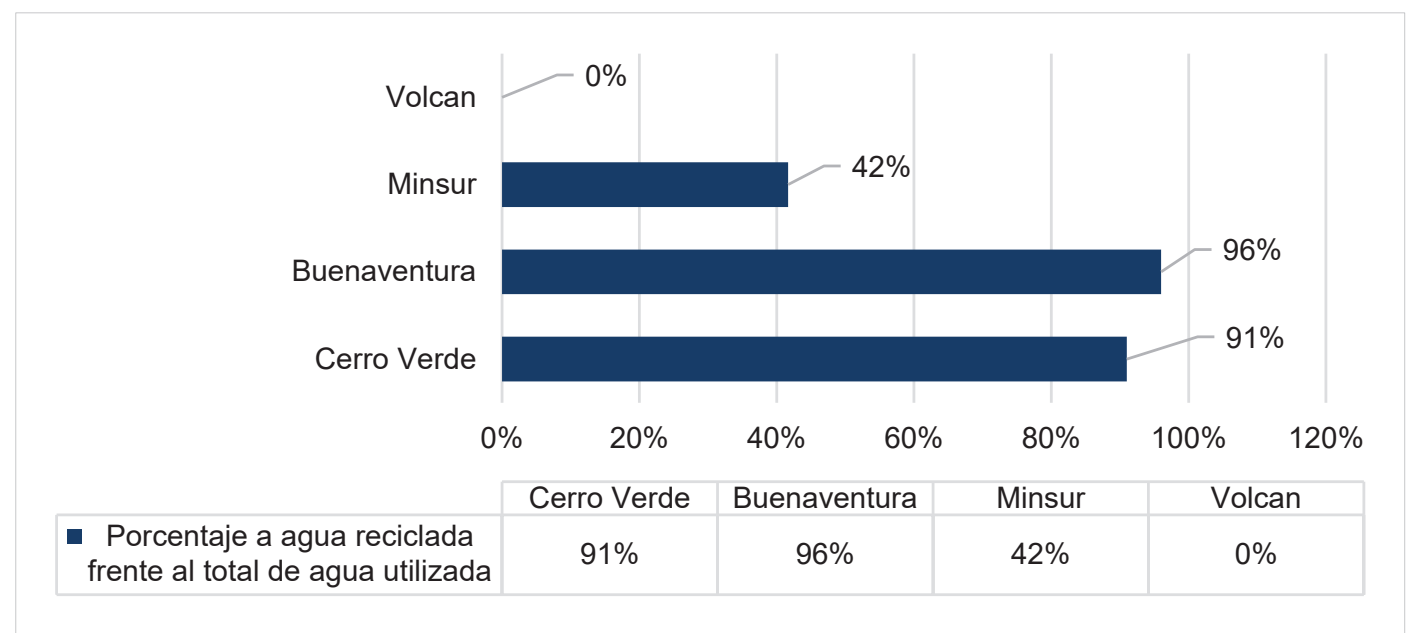

Figura 3. Porcentaje de agua reutilizada por las empresas mineras analizadas.

Fuente: Reportes de sostenibilidad de Cerro Verde, Buenaventura, Minsur y Volcan. Database GRI, 2018 (https://database.globalreporting.org/)

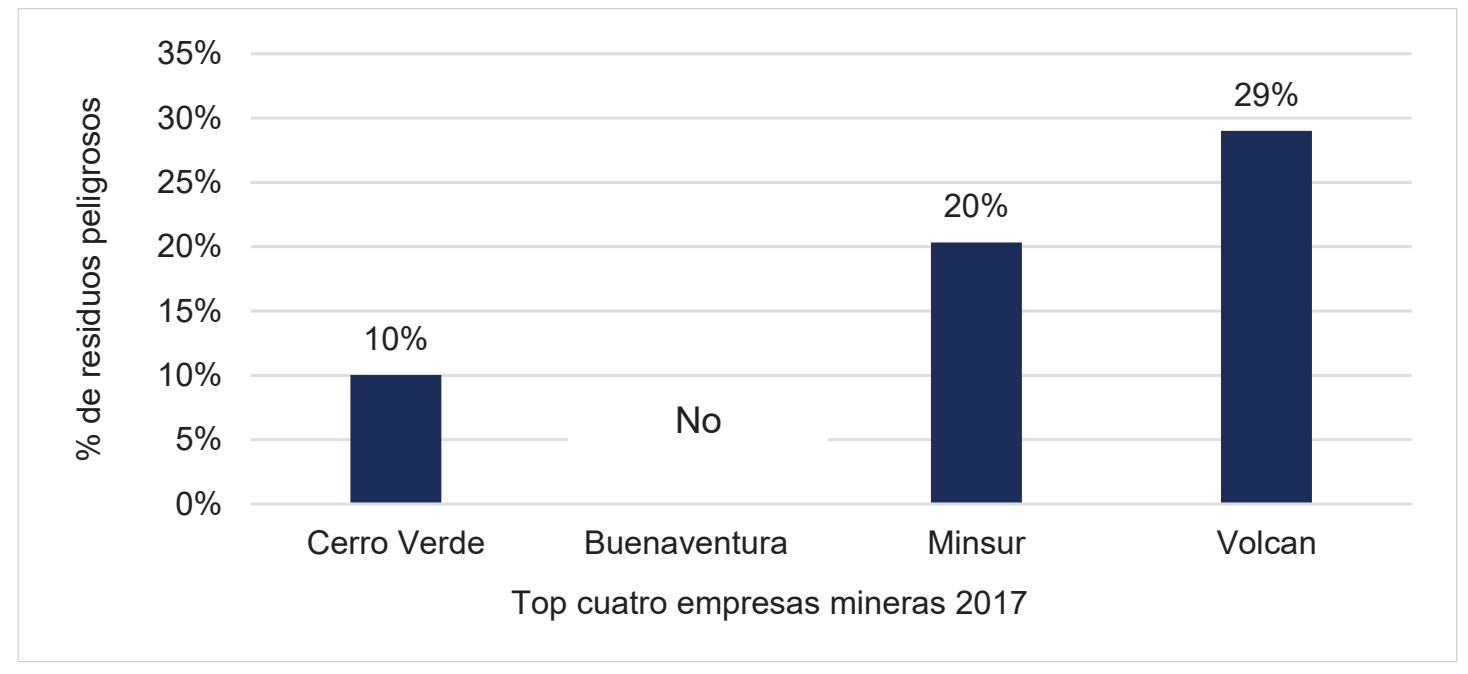

Figura 4. Porcentaje de residuos peligrosos frente al total de residuos.

Fuente: Reportes de sostenibilidad de Cerro Verde, Buenaventura, Minsur y Volcan. Database GRI, 2018

(https://database.globalreporting.org/)

Indicador 6: Porcentaje de residuos peligrosos frente al total de residuos

Según el Anuario Minero del Minem (2017), existe la necesidad de mejorar la información proporcionada por las empresas mineras en relación con los residuos peligrosos. En esa línea, la información sobre residuos peligrosos es relevante para los stakeholders y, por lo tanto, contribuye a la generación de valor a estos (Ministerio de Energía y Minas, 2017). Por lo mencionado anteriormente, se propone el siguiente indicador:
Fórmula 6: Porcentaje de residuos peligrosos frente al total de residuos

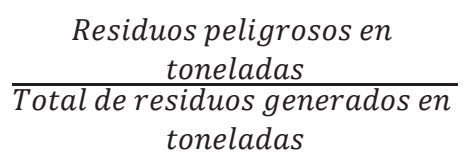

La Figura 4 evidencia que Buenaventura ha optado por no comunicar sobre los residuos peligrosos que se generan a partir de sus actividades. Por otro lado, las empresas mineras reportaron información sobre residuos peligrosos, lo cual permitió calcular el indicador para el resto de las 
empresas: Cerro Verde, Minsur y Volcán con 10\%, 20\% y $29 \%$, respectivamente. En general, se puede ver que estas tres mineras generan valor a sus stakeholders, a diferencia de Buenaventura, ya que la empresa no está cumpliendo con comunicar un tema relevante al sector minero. Por último, Cerro Verde es la organización con un indicador más favorable, debido a que muestra la capacidad de manejar los residuos peligrosos de manera efectiva.

Luego del análisis descriptivo, se plantearon y calcularon seis indicadores a partir de informes técnicos, como el Anuario Minero del Ministerio de Energía y Minas (2017), el Reporte de conflictos sociales de la Defensoría del Pueblo (2017), el estudio sobre la gestión social en la industria minera peruana -desarrollado por Brereton et al. (2018) y los reportes de sostenibilidad elaborados con la metodología GRI de las empresas examinadas. En tal sentido, los indicadores propuestos pertenecen a distintos temas vinculados a la dimensión social que son relevantes para las empresas mineras como: los accidentes laborales, los mecanismos de participación ciudadana la capacitación del recurso humano, canon minero. Del mismo modo, se presentaron indicadores ambientales pertenecientes a los temas de reciclaje de agua y residuos peligrosos.

\section{DISCUSIÓN}

Entre los numerosos estudios vinculados con los Reportes de Sostenibilidad (RS) destacan las investigaciones de Briano (2014), Correa et al. (2016) y Hernández (2016). Por un lado, Briano (2014) analizó los indicadores de los RS de empresas de países latinoamericanos, cuya selección se basó en el grado de liquidez y capitalización. Por otro lado, Correa et al. (2016) y Hernández (2016) analizaron los RS de las principales empresas latinoamericanas de diferentes sectores, como el financiero, comercial e industrial. Cabe precisar que ningún estudio examinado se centró en el desarrollo de indicadores complementarios relativos a las dimensiones social y ambiental de empresas del sector minero, enfoque que fue abordado en el presente estudio. Hasta el 2017, el sector minero presentó un incremento de los conflictos socioambientales con respecto a los años anteriores (Defensoría del Pueblo, 2017); ello podría deberse al hecho de que las empresas mineras peruanas utilizan indicadores sociales y ambientales potencialmente irrelevantes. El estudio sugiere el uso de los seis indicadores complementarios propuestos correspondientes a las dimensiones social y ambiental. Ficco et al. (2017) sostienen que uno de los principales beneficios de comunicar indicadores de tal índole es el fomento de la sostenibilidad de las empresas mineras a largo plazo, aspecto crítico del sector (KPMG, 2017). Asimismo, García y Larrán (2004) y Robbins y Coulter (2009) indican que la comunicación de dichos indicadores puede: reducir el costo de capital, al disminuir la incertidumbre; incrementar los precios por acción; mejorar la imagen pública; y contribuir a la disminución de los costos legales, como de los litigios. Por ello, utilizar los indicadores complementarios propuestos en el presente estudio favorecería la sostenibilidad de las empresas mineras y el desarrollo del sector, así cómo la creación de mayor valor para sus colaboradores, accionistas, acreedores y otros grupos de interés.

\section{REFERENCIAS}

Alfaro, C., y Gómez, J. (2016). Un sistema de indicadores para la medición, evaluación, innovación y participación orientado a la administración pública. Revista de ciencias sociales, 4(12), 274-290.

Brereton, D., Cano, Á., y Paredes, Á. (2018). Prácticas de gestión social en la industria minera peruana: hallazgos clave de la encuesta a empresas de la Sociedad Nacional de Minería, Petróleo y Energía (SNMPE). Recuperado del sitio de Internet de la Universidad del Pacífico. https://www.up.edu.pe/SiteAssets/Lists/Prensa\%20 Noticias/AllItems/Brereton_Cano_Paredes_Practicas\%20de\%20Gestion\%20Social\%20Industria\%20Minera\%20Peruana\%202018.pdf

Briano, G. (2014). Factores que inciden en una mayor transparencia de gobernanza corporativa en empresas cotizadas latinoamericanas. Revista Mexicana de Economía y Finanzas, 9(2), 105-124.

Correa, J. A., Hernández, M. C., Vásquez, L., y Soto, Y. M. (2016). Reportes integrados y generación de valor en empresas colombianas incluidas en el Índice de Sostenibilidad Dow Jones. Cuadernos de Contabilidad, 17(43), 73-108.

DATABASE GRI. (2018). GRI Sustainability Disclosure Database. Recuperado de: https://database.globalreporting.org/

Defensoría del Pueblo. (2017). Reporte de conflictos sociales $N^{\circ} 166$. Recuperado de: https://www. defensoria. gob.pe/temas.php.

Ficco, C. R., García, G. A., y Valenzuela, J. L. (Julio de 2017). Información sobre capital intelectual e informes de sostenibilidad. Un estudio empírico en el mercado de capitales argentino. Revista Visión Contable, (16), 50-72.

García, E. y Larrán, J. (2004). La relevancia de la información no financiera en la estrategia empresarial de divulgación voluntaria: percepciones empresa-analista sobre su utilidad. Revista valenciana de economía y hacienda, (12), 127-148. 
Guevara, M. (2015). La importancia de prevenir los riesgos laborales en una organización. Bogotá: Universidad Militar Nueva Granada.

Hernández, J. (2016). Estado de la Situación de la información de Sostenibilidad de las Empresas Peruanas. Revista Contabilidad \& Sistemas, 9, 47-55.

KPMG. (2017). The KPMG Survey of Corporate Responsiblity Reporting. Recuperado de: https://assets.kpmg/ content/dam/kpmg/xx/pdf/2017/10/kpmg-survey-of-corporate-responsibility-reporting-2017.pdf

Ministerio de Energía y Minas. (2017). Anuario Minero 2017. Perú. Recuperado de: https://cdn.www.gob.pe/ uploads/document/file/98805/ANUARIO_MINERO_2017_1_.pdf

Najmaei, A. y Sadeghinejad, Z. (2014). Business Model Theory and Research: A Philosophical Discussion. 3 Entrepreneurship, Start-ups and Small Business, 1-21.

Nicomedes, E. (2018). Utilidad de la Nueva Epistemología, según Mario Bunge. Lima: Repositorio Universidad Santo Domingo de Guzmán.

Organización Internacional del Trabajo. (2016). Mejore su negocio. El recurso humano y la productividad. Ginebra: Autor.

PricewaterhouseCoopers. (2019). Mine 2019: Generando recursos para el futuro. Perú. Recuperado de: https:// www.pwc.pe/es/publicaciones/assets/brochures/ mine2019_pwc.pdf
Programa de las Naciones Unidas para el Desarrollo. (2014). Informe Regional del Desarrollo Humano. Nueva York: ONU.

Robbins, S. P., y Coulter, R. M. (2009). Responsabilidad social y ética administrativa (10a ed.). Pearson.

Salim, C. (2014). Analysis of work related accidents in the mining sector 2000-10: the case of Minas Gerais State, Brazil. Occupational \& Environmental Medicine, 71(1), A79-A79.

Stanford Encyclopedia of Philosophy. (2013). Pragmatism. Obtenido de https://plato.stanford.edu/entries/pragmatism/\#PraMax

Striedinger, J. (2014). Responsabilidad social para las pyme sector calzado bajo las normas ISO 26000 (Tesis de grado, Universidad Militar Nueva Granada, Colombia). Recuperada de: https://repository.unimilitar.edu. co/bitstream/handle/10654/12359/RESPONSAB?sequence $=2$

Velarde, P. (2018). Violencia y Polarización en la Conflictividad Minera Peruana: Las Bambas (Tesis de licenciatura). Pontifica Universidad Católica del Perú. Recuperado de: http://tesis.pucp.edu.pe/repositorio/ handle/20.500.12404/12261 